\title{
Atuação do fisioterapeuta mediante a pandemia da covid-19 em um hospital de referência no interior da Amazônia Legal
}

\author{
Physiotherapist's performance through the covid-19 pandemic in a referral hospital in the \\ interior of the Legal Amazon
}
Actuación del fisioterapeuta ante la pandemia del covid-19 en un hospital de referencia en el interior de la Amazonia Legal

Elenir Silva de Carvalho ${ }^{1 *}$, Alana Kundsin ${ }^{1}$.

\begin{abstract}
RESUMO
Objetivo: Observar a atuação do fisioterapeuta no âmbito hospitalar perante a assistência aos pacientes acometidos pela COVID-19, em um hospital de referência no interior da Amazônia Legal. Métodos: Pesquisa transversal de caráter descritivo, exploratório, com abordagem quantitativa. Os dados foram coletados através de um questionário online, com a participação dos profissionais da área de fisioterapia, a coleta consistiu por um período de um mês em um hospital público do interior de Rondônia. Resultados: Foi possível constatar a presença de profissionais jovens, com predominância de atuação em unidade de terapia intensiva com carga horária de $24 \mathrm{~h} /$ dia. Devido a pandemia da covid-19, foi observado a contratação de profissionais emergenciais. Os fisioterapeutas desta instituição, 96,7\% relataram que receberam treinamentos para 0 manejo dos pacientes infectados por SAR-CoV-2 e 33,3\% destes profissionais já sofreram infecção por este vírus. Conclusão: $O$ fisioterapeuta vem atuando na condução do suporte ventilatório invasivo e não invasivo, manejo da oxigenoterapia e da posição prona, realizando exercícios com ênfase na otimização e reabilitação da função motora e respiratória, auxiliando em procedimentos corriqueiros dos cuidados intensivos como a intubação orotraqueal, extubação, reanimação cardiopulmonar.
\end{abstract}

Palavras-chave: Fisioterapia, COVID-19, Unidades de terapia intensiva, Unidade hospitalar de saúde pública.

\begin{abstract}
Objective: Observe the performance of the physiotherapist in the hospital environment when assisting patients affected by COVID-19, in a reference hospital in the interior of the Legal Amazon. Methods: Cross-sectional, descriptive, exploratory research with a quantitative approach. The data were collected through an online questionnaire, with the participation of physiotherapy professionals, the collection consisted of a period of one month in a public hospital in the interior of Rondônia. Results: It was possible to verify the presence of young professionals, with a predominance of work in an intensive care unit with a workload of 24 hours / day. Due to the covid-19 pandemic, the hiring of emergency professionals was observed. The physiotherapists at this institution, $96.7 \%$ reported that they received training in the management of patients infected with SAR-CoV2 and $33.3 \%$ of these professionals have already suffered infection with this virus. Conclusion: The physiotherapist has been active in conducting invasive and non-invasive ventilatory support, handling oxygen therapy and the prone position, performing exercises with an emphasis on optimizing and recovering motor and respiratory function, assisting in routine intensive care procedures such as orotracheal intubation, extubation, cardiopulmonary resuscitation.
\end{abstract}

Key words: Physiotherapy, COVID-19, Intensive care units, Hospital public health unit.

${ }^{1}$ Hospital Regional de Cacoal (HRC), Cacoal - RO. *E-mail: elenirpvh@hotmail.com

SUBMETIDO EM: 1/2021

ACEITO EM: 2/2021

PUBLICADO EM: 2/2021 


\section{RESUMEN}

Objetivo: Observar el desempeño del fisioterapeuta en el ámbito hospitalario al atender a pacientes afectados por COVID-19, en un hospital de referencia en el interior de la Amazonia Legal. Métodos: Investigación transversal, descriptiva, exploratoria con enfoque cuantitativo. Los datos fueron recolectados a través de un cuestionario en línea, con la participación de profesionales en el campo de la fisioterapia, la recolección consistió en un período de un mes en un hospital público del interior de Rondônia. Resultados: se pudo constatar la presencia de profesionales jóvenes, con predominio del trabajo en una unidad de cuidados intensivos con una carga de trabajo de 24 horas / día. Debido a la pandemia de covid-19, se observó la contratación de profesionales de emergencia. Los fisioterapeutas de esta institución, el 96,7\% refirieron haber recibido formación en el manejo de pacientes infectados por SAR-CoV-2 y el 33,3\% de estos profesionales ya han padecido infección por este virus. Conclusión: El fisioterapeuta ha estado activo en la realización de soporte ventilatorio invasivo y no invasivo, manejo de oxigenoterapia y decúbito prono, realizando ejercicios con énfasis en la optimización y recuperación de la función motora y respiratoria, asistiendo en procedimientos rutinarios de cuidados intensivos como intubación orotraqueal extubación, reanimación cardiopulmonar.

Palabras clave: Fisioterapia, COVID-19, Unidades de cuidados intensivos, Unidad hospitalaria de salud pública.

\section{INTRODUÇÃO}

Em dezembro de 2019, sucedeu-se diversos casos de pneumonia de etiologia desconhecida, em Wuhan, China. Logo em seguida, foi iniciado pesquisas etiológicas sendo encontrado um novo coronavírus como o causador do surto, sendo denominado inicialmente pela Organização Mundial da Saúde (OMS) como o novo coronavírus de 2019 (2019-nCoV), em 11 de fevereiro de 2020 a OMS oficializou a denominação do vírus como a doença do coronavírus 2019 (COVID-19), e o grupo de estudo para coronavírus do Comitê Internacional de Taxonomia de Vírus através da filogenia e taxonomia designou como coronavírus 2 da síndrome respiratória aguda grave (SARS-CoV-2). A doença propagou-se pelo mundo rapidamente sendo declarado uma pandemia pela OMS em 11 de março de 2020 (GORBALENYA AE, et al., 2020; SUN P, et al., 2020; BRASIL, 2020).

O vírus SARS-Cov-2 é o responsável pela nova doença que acomete o mundo, altamente transmissível, disseminado através de gotículas e aerossóis produzidos pela fala, espirro, tosse, secreção entre outros. Além do contato direto com a pele de indivíduos ou superfícies contaminadas (fômites) seguido por contato em face do indivíduo ainda sadio. Segundo a OMS, o vírus possui período de incubação variante de 1 a 14 dias, com média de 5-7 dias. Os infectados podem ser assintomáticos ou sintomáticos. No último caso podem apresentar os principais sintomas: febre, tosse, dispneia, mialgia, fadiga, fraqueza, êmese, diarreia, anosmia, ageusia (WIERSINGA WJ, et al., 2020; BRASIL, 2020).

Segundo Wang F, et al. (2020) o SARS-CoV-2 pode acometer o sistema respiratório e/ou neurológico, tendo, como os acometimentos mais frequentes as alterações nas vias do sistema respiratório sendo via superior e/ou inferior. Cerca de $80 \%$ dos infectados por COVID-19 evoluem para manifestações clínicas leves, entretanto, nos casos mais graves podem evoluir com insuficiência respiratória aguda e até mesmo ao óbito. A piora clínica dos pacientes infectados podem ocorrer de forma abrupta, aproximadamente $15 \%$ dos infectados possuem a necessidade de internação hospitalar devido a hipoxemia e $5 \%$ necessitam de cuidados em unidade de terapia intensiva (UTI) (BRASIL, 2020; NORONHA KVMS et al., 2020).

Os casos de sintomas leves são mantidos em tratamento e monitorização domiciliar. Nos casos que evoluem com necessidade de oxigenoterapia suplementar e suporte ventilatório não invasivo ou invasivo, são encaminhados para internação hospitalar. São considerados fatores de risco, os indivíduos com uma ou mais comorbidades prévias, tais como, a diabetes, hipertensão, obesidade, idade avançada, doença pulmonar obstrutiva crônica, asma, neoplasia, imunodepressão, gestação de alto risco, doenças cromossômicas com déficit imunológico, miocardiopatias, entre outras doenças. Devido a propagação em alta velocidade pelo mundo e a rápida piora clínica dos indivíduos infectados, os hospitais apresentaram uma alta taxa de 
internações, levando a sua ocupação máxima (BRASIL, 2020; GRASSELLI G, et al., 2020; NORONHA KVMS, et al.,2020).

Atualmente, ainda não possui um tratamento específico, entretanto, vacinas se encontram em fases de estudos para o combate do vírus, como por exemplo: a vacina coronavac, que recentemente foi divulgado o resultado da fase 3 do estudo produzido pela SINOVAC com parceria pelo Instituto Butantan, mostrando eficácia de $100 \%$ nos casos moderados e graves e $78 \%$ de eficácia nos casos considerados leves (INSTITUTO BUTANTAN, 2021).

A UTI é um ambiente desenvolvido para os pacientes que necessitam de cuidados ininterruptos, através de cuidados intensivos de uma equipe multiprofissional. Nas últimas décadas, as UTI's têm ganhado avanços científicos e tecnológicos que proporcionam um aumento da sobrevida, entretanto, é possível observar longas estadias de pacientes. Estas internações prolongadas interferem na funcionalidade do sistema respiratório e osteomuscular gerando impactos negativos importantes na qualidade de vida, durante a estadia hospitalar e após a alta. No intuito de promover monitorização, prevenção e reabilitação da função motora e respiratória destes pacientes, destaca-se a atuação dos profissionais de fisioterapia, que visam a prevenção e reabilitação do sistema cardiorrespiratório, neuromuscular e musculoesquelético através de mobilizações precoces, manejos de intervenções preventivas e reabilitadora de todo o corpo humano (MATOS CA, et al., 2016).

Assim, como os demais pacientes de internações prolongadas dentro das UTI's, os indivíduos acometidos pelo vírus SAR-CoV-2, possuem fatores de risco para desenvolverem a fraqueza muscular adquirida de unidade de terapia intensiva e o declínio da funcionalidade (MARTINEZ BP, ANDRADE FMD, 2020).

Os profissionais da área de saúde são expostos diariamente ao risco de contaminação pelo vírus da COVID-19, pois atuam em ambientes contaminados. Ainda estão sujeitos a sofrerem com a falta de recursos humanos e equipamentos de proteção individual adequados gerando um aumento do stress físico e mental (SANTANA G, et al., 2020). O fisioterapeuta é um dos profissionais atuantes na linha de frente do enfretamento do coronavírus 2019, que avalia, prescreve e trata as alterações cinéticas funcionais, assim, como as alterações respiratórias (GUIMARÃES F, 2020).

Diante deste contexto e a escassez de estudos sobre o enfrentamento dos fisioterapeutas diante da COVID-19, o objetivo desta pesquisa foi analisar a atuação do fisioterapeuta no âmbito hospitalar perante a assistência aos pacientes acometidos pela COVID-19, em um hospital de referência no interior da Amazônia Legal.

\section{MÉTODOS}

Trata-se de uma pesquisa do tipo transversal de caráter descritivo, exploratório, com abordagem quantitativa com dados coletados através de um questionário online. A pesquisa ocorreu em um hospital público do interior de Rondônia (RO), composta pela amostra de 30 profissionais de fisioterapia atuantes no atendimento hospitalar dos setores exclusivos para o tratamento dos infectados por SAR-CoV-2. O hospital é uma instituição pública, que realiza atendimentos de média e alta complexidade, é macrorregião de saúde do estado e atualmente um hospital de referência no tratamento da COVID-19.

O questionário online foi aplicado através da ferramenta Google Forms, composto por 26 questões fechadas, desenvolvido pelos pesquisadores. A coleta ocorreu no período de 30 dias (novembro/2020), tendo dados coletados relacionados a idade, gênero, perfil de nível de formação e condutas aplicadas no atendimento ao paciente COVID-19, de acordo com as orientações descritas sobre a atuação do fisioterapeuta e o manejo dos pacientes infectados pela COVID-19 pela Associação Brasileira de Fisioterapia Cardiorrespiratória e Fisioterapia em Terapia Intensiva (ASSOBRAFIR).

Inicialmente, a pesquisa foi explicada para a coordenação de fisioterapia da instituição, respeitando as medidas preventivas, conforme o DECRETO № 25.470, DE 21 DE OUTUBRO DE 2020 no âmbito do estado de $\mathrm{RO}$, onde foi obrigatório o uso de máscara, manutenção do distanciamento mínimo de 2 metros e higienização das mãos sempre que necessário. Em seguida, a pesquisa foi explicada aos colaboradores 
fisioterapeutas e feito convite online para participação. Os profissionais que quiseram participar acessaram link encaminhado com redirecionamento ao questionário eletrônico.

Por se tratar de uma pesquisa online devido a pandemia da COVID-19, a assinatura do Termo de Consentimento Livre e Esclarecido (TCLE) ocorreu por meio eletrônico, na abertura do questionário. Cada participante que declarou que leu e aceitou participar, recebeu uma cópia do TCLE, enviado através do seu endereço eletrônico descrito na abertura do questionário.

Os dados foram processados de forma descritiva, sendo utilizado frequência e porcentagem, apresentados em tabelas e gráfico, foram consolidados através da análise dos programas SPSS Statistic versão 2.0 e Microsoft Office Word. Posteriormente analisados e discutidos.

A pesquisa foi realizada após a aprovação do Comitê de Ética em Pesquisa (CEP) da Universidade Federal de Rondônia (UNIR), por meio da CAAE 38663420.0.0000.5300 e do parecer 4.397.816, bem como a autorização da direção do hospital. Seguindo as normas da Resolução 466/2012 do Conselho Nacional de Saúde (CNS).

\section{RESULTADOS}

A amostra foi composta por 30 fisioterapeutas sendo $73,3 \%$ do gênero masculino e $26,7 \%$ do gênero feminino. As faixas etárias foram fracionadas: 20 a 30 anos correspondente a 53,3\% dos profissionais, 31 a 40 anos $40 \%$ e 41 a 50 anos $6,7 \%$ profissionais. Foi verificado que nenhum possui formação acadêmica com período inferior a 1 ano, um total de 33,3\% possui período de formação de 1 a 2 anos, 13,3\% possuem um período de 3 a 4 anos, 23,4\% possuem período de formação entre 5 a 7 anos, 13,3\% possuem período de formação de 8 a 10 anos e 16,7\% profissionais possuem formação em período superior a 10 anos. Em relação ao grau acadêmico, 33,3\% indivíduos mencionaram possuir o título de graduação em fisioterapia, 63,4\% indivíduos responderam ser especialistas e 3,3\% responderam ser mestre, não há nesta amostra profissionais que possuem o nível de doutorado e pós-doutorado.

No contexto da distribuição e caracterização dos fisioterapeutas, $80 \%$ dos profissionais são atuantes somente em UTI e $20 \%$ atuantes em ambos os setores de UTI e enfermaria. Verifica-se que $6,7 \%$ dos indivíduos realizam plantões com carga horária menor que 12 horas, 16,6\% plantões de 12 horas e 76,7\% realizam plantões de 24 horas, sendo que $10 \%$ realizam plantões no período diurno e $16,7 \%$ entre os períodos diurnos e/ou noturnos. Na UTI 16,7\% realizam plantões diurnos e $83,3 \%$ em ambos os períodos diurnos/noturnos (Tabela 1).

Os vínculos empregatícios destes fisioterapeutas são distribuídos em três classes: $33,3 \%$ servidores estatutários, $50 \%$ de contrato emergencial e $16,7 \%$ do programa de residência multiprofissional em cuidados intensivos. Destes profissionais, $2 \%$ não possuíam experiência em cuidados com o paciente crítico, $20 \%$ relataram ter experiência menor que 6 meses, 13,3\% responderam possuir experiência de 6 meses a 1 ano, $26,7 \%$ possuem experiência de 1 a 2 anos, 23,3\% possuem experiência de 3 a 6 anos, $6,7 \%$ possuem experiência de 7 a 10 anos e 6,7\% relataram possuir um período maior que 10 anos de experiência. 
Tabela 1 - Distribuição e caracterização dos profissionais da área de fisioterapia.

\begin{tabular}{|c|c|c|}
\hline Variáveis & $\mathrm{N}=\mathbf{3 0}$ & $\%$ \\
\hline \multicolumn{3}{|l|}{ Setor } \\
\hline UTI & 24 & 80 \\
\hline UTI e enfermaria & 6 & 20 \\
\hline \multicolumn{3}{|l|}{ Carga horária } \\
\hline$<12$ horas & 2 & 6,7 \\
\hline 12 horas & 5 & 16,6 \\
\hline 24 horas & 23 & 76,7 \\
\hline \multicolumn{3}{|l|}{ Profissional } \\
\hline Servidor estatutário & 10 & 33,3 \\
\hline Contrato emergencial & 15 & 50 \\
\hline Programa de residência multiprofissional & 5 & 16,7 \\
\hline \multicolumn{3}{|l|}{ Experiência com pacientes críticos } \\
\hline Sim & 28 & 93,3 \\
\hline Não & 2 & 6,7 \\
\hline \multicolumn{3}{|c|}{ Tempo de experiência com pacientes críticos } \\
\hline$<6$ meses & 6 & 20 \\
\hline 6 meses -1 ano & 4 & 13,3 \\
\hline 1 ano -2 anos & 8 & 26,7 \\
\hline $3-6$ anos & 7 & 23,3 \\
\hline $7-10$ anos & 2 & 6,7 \\
\hline$>10$ anos & 2 & 6,7 \\
\hline Não respondeu & 1 & 3,3 \\
\hline \multicolumn{3}{|l|}{ Na enfermaria o plantão é? } \\
\hline Diurno & 3 & 10 \\
\hline Diurno e/ou noturno & 5 & 16,7 \\
\hline Nenhum & 22 & 73,3 \\
\hline \multicolumn{3}{|l|}{ Na UTI o plantão é? } \\
\hline Diurno & 5 & 16,7 \\
\hline Diurno e/ou noturno & 25 & 83,3 \\
\hline
\end{tabular}

Fonte: Carvalho ES e Kundsin A, 2020.

Sobre a utilização da posição prona como um dos recursos para o tratamento, 96,7\% já utilizaram e 3,3\% não. Quanto a experiência prévia a pandemia com a prona, 36,7\% responderam que possuíam experiência e $63,3 \%$ não. No quesito receber treinamentos para a o enfrentamento da covid-19, $96,7 \%$ dos profissionais relataram que receberam e 3,3\% não. A instituição forneceu diversos treinamentos: o manejo da ventilação mecânica invasiva (VMI), onde 83,3\% receberam e 16,7\% não; o manejo da ventilação não invasiva (VNI), onde $56,7 \%$ relataram que receberam e $43,3 \%$ não; manejo da posição prona, onde $86,7 \%$ responderam que receberam e 13,3\% não; o manejo da oxigenoterapia, onde $46,7 \%$ relataram receber o treinamento e 53,3\% não; treinamento sobre a utilização de equipamento de proteção individual (EPI's), onde $90 \%$ relataram que receberam e $10 \%$ não; treinamento sobre a utilização de escalas funcionais, onde $10 \%$ receberam e 90\% não; treinamentos sobre os recursos para manutenção e reabilitação da função motora e da função respiratória, onde 13,3\% relataram que receberam, isto em ambos os treinamentos dos recursos; além destes treinamentos, 6,7\% responderam que receberam outros treinamentos, aos quais não foram descritos. Os fisioterapeutas foram questionados se realizam avaliação dos músculos periféricos, 53,3\% responderam que realizam e $46,7 \%$ não. Com relação as estratégias fisioterapêuticas utilizadas para a otimização e reabilitação da função motora, foi dada alternativa de realizar somente de mobilização passiva onde $13,3 \%$ responderam que sim e $86,7 \%$ não. No quesito mobilização articular $93,3 \%$ responderam que realizam conforme a avaliação individualizada podendo ser: passiva, ativa-assistida ou ativa e $6,7 \%$ relataram que não utilizam. Sobre a cicloergometria, $86,7 \%$ relataram que utilizam deste recurso e $13,3 \%$ não. A estimulação elétrica neuromuscular é utilizada por $16,7 \%$ e não utilizada por $83,3 \%$. Sobre o ortostatismo, $80 \%$ dos participantes utilizam e $20 \%$ não. A sedestação no leito é efetuada por $93,7 \%$ e $6,7 \%$ não. Quanto a sedestação fora do leito $86,7 \%$ responderam que realizam e $13,3 \%$ não. Foi questionado se esses profissionais utilizam outras 
condutas fisioterapêuticas para otimização e reabilitação da função motora, $80 \%$ responderam que não e $20 \%$ responderam que utilizam de outras condutas, porém não foram especificadas quais (Tabela 2).

Tabela 2 - Distribuição das frequências relativas e absolutas com ênfase na assistência fisioterapêutica e contaminação.

\begin{tabular}{|c|c|c|c|c|}
\hline \multirow{2}{*}{ Variáveis } & \multicolumn{2}{|c|}{ Sim } & \multicolumn{2}{|c|}{ Não } \\
\hline & $\mathbf{N}$ & $\%$ & $\mathbf{N}$ & $\%$ \\
\hline Utilização da posição prona junto à equipe na assistência ao paciente & 29 & 96,7 & 1 & 3,3 \\
\hline Experiência prévia a pandemia em relação a posição prona & 11 & 36,7 & 19 & 63,3 \\
\hline $\begin{array}{l}\text { Recebeu algum tipo de treinamento para o manejo do paciente com COVID- } \\
19 \text { pela instituição }\end{array}$ & 29 & 96,7 & 1 & 3,3 \\
\hline Treinamento sobre o manejo da VMI & 25 & 83,3 & 5 & 16,7 \\
\hline Treinamento sobre o manejo da VNI & 17 & 56,7 & 13 & 43,3 \\
\hline Treinamento sobre a posição prona & 26 & 86,7 & 4 & 13,3 \\
\hline Treinamento sobre de oxigenoterapia & 14 & 46,7 & 16 & 53,3 \\
\hline Treinamento sobre a utilização de EPl's & 27 & 90 & 3 & 10 \\
\hline Treinamento sobre a utilização de escalas funcionais & 3 & 10 & 27 & 90 \\
\hline $\begin{array}{l}\text { Treinamento sobre os recursos para manutenção/reabilitação da função } \\
\text { respiratória }\end{array}$ & 4 & 13,3 & 26 & 86,7 \\
\hline $\begin{array}{l}\text { Treinamento sobre os recursos para manutenção/reabilitação da função } \\
\text { motora }\end{array}$ & 4 & 13,3 & 26 & 86,7 \\
\hline Outros treinamentos para manutenção/reabilitação da função motora & 2 & 6,7 & 28 & 93,3 \\
\hline $\begin{array}{l}\text { Realização da avaliação dos músculos periféricos para otimizar/reabilitar a } \\
\text { função motora }\end{array}$ & 16 & 53,3 & 14 & 46,7 \\
\hline $\begin{array}{l}\text { Realização isolada de mobilização passiva, para otimizar/reabilitar a função } \\
\text { motora }\end{array}$ & 4 & 13,3 & 26 & 86,7 \\
\hline $\begin{array}{l}\text { Realização de mobilização articular, podendo ser: passiva, ativo - assistido, } \\
\text { ativo para otimizar/reabilitar a função motora }\end{array}$ & 28 & 93,3 & 2 & 6,7 \\
\hline Utilização de cicloergômetro para otimizar/reabilitar a função motora & 26 & 86,7 & 4 & 13,3 \\
\hline $\begin{array}{l}\text { Realização de estimulação elétrica neuromuscular para otimizar/reabilitar a } \\
\text { função motora }\end{array}$ & 5 & 16,7 & 25 & 83,3 \\
\hline Realização de ortostatismo para otimizar/reabilitar a função motora & 24 & 80 & 6 & 20 \\
\hline Realização no leito para otimizar/reabilitar a função motora & 28 & 93,3 & 2 & 6,7 \\
\hline $\begin{array}{l}\text { Realização de sedestação fora do leito para otimizar/reabilitar a função } \\
\text { motora }\end{array}$ & 26 & 86,7 & 4 & 13,3 \\
\hline ção de outras condutas para otimizar/reabilitar a função motora & 6 & 20 & 24 & 80 \\
\hline Realização de VNI para otimizar/reabilitar a função respiratória & 30 & 100 & 0 & 0 \\
\hline $\begin{array}{l}\text { Realização de mobilização associada ao uso de incentivador respiratório } \\
\text { para otimizar/reabilitar a função respiratória }\end{array}$ & 16 & 53,3 & 14 & 46,7 \\
\hline $\begin{array}{l}\text { Realização de estimula elétrica neuromuscular para otimizar/reabilitar a } \\
\text { função respiratória }\end{array}$ & 3 & 10 & 27 & 90 \\
\hline $\begin{array}{l}\text { Realização de sedestação no leito para otimizar/reabilitar a função } \\
\text { respiratória }\end{array}$ & 22 & 73,3 & 8 & 26,7 \\
\hline $\begin{array}{l}\text { Realização de sedestação fora do leito para otimizar/reabilitar a função } \\
\text { respiratória }\end{array}$ & 20 & 66,7 & 10 & 33,3 \\
\hline Realização de ortostatismo para otimizar/reabilitar a função respiratória & 16 & 53,3 & 14 & 46,7 \\
\hline Realização de outras condutas para otimizar/reabilitar a função respiratória & 6 & 20 & 24 & 80 \\
\hline Testaram positivo para COVID-19 & 10 & 33,3 & 20 & 66,7 \\
\hline
\end{tabular}

Fonte: Carvalho ES e Kundsin A, 2020.

No tocante de otimização e reabilitação da função respiratória, $100 \%$ responderam que fazem o uso da VNI como um recurso, 53,3\% utilizam a mobilização associada com a utilização de incentivador respiratório e $46,7 \%$ não realizam essa associação. O uso da estimulação elétrica neuromuscular é utilizado por $10 \%$ dos profissionais e $90 \%$ não. Em relação a sedestação no leito $73,3 \%$ relataram realizar e $26,7 \%$ não. Sobre sedestação fora do leito $66,7 \%$ responderam que realizam e $33,3 \%$ não. O ortostatismo é realizado por $53,3 \%$ dos fisioterapeutas e $46,7 \%$ não. Dos participantes $20 \%$ relataram que desfrutam de outras condutas que não 
foram especificadas e $80 \%$ não se utiliza de outras condutas. Com relação a contaminação da covid-19, $33,3 \%$ relataram ter testado positivo até o término da coleta de dados e $66,7 \%$ relataram que não haviam se contaminado.

No que diz respeito, a frequência do atendimento da fisioterapia com ênfase na função motora, $66,7 \%$ dos profissionais responderam que realizam com frequência tal atendimento, 33,3\% realizam às vezes e não houve dados para a não realização. Os profissionais que relataram realizar às vezes, os motivos foram: a falta de EPI, equipamentos, equipe sobrecarregada devido o nível de complexidade clínica dos pacientes, falta de recursos humanos e outros motivos que não foram descritos (Tabela 3) (Gráfico 1). Sobre a utilização de protocolo de mobilização precoce neste perfil de paciente, $70 \%$ relataram que utilizam, $6,7 \%$ não e $23,3 \%$ relataram não conhecer nenhum protocolo (Tabela 4).

Em relação, a frequência ao atendimento da fisioterapia voltada para a função respiratória, 96,7\% responderam que realizam com frequência este atendimento fisioterapêutico, 3,3\% realizam às vezes, e não houve relatos da não realização. Os profissionais que realizam às vezes, os motivos para tal posicionamento são: falta de equipamento, protocolos para este perfil de doença e equipe sobrecarregada devido o nível de complexidade clínica dos pacientes. Durante o procedimento de intubação orotraqueal de um paciente, $96,7 \%$ relataram que participam e 3,3\% participam às vezes, não foi obtido dados de profissionais que não participam. No quesito recebimento de EPI's adequados ao atendimento dos pacientes com COVID 19, 60\% responderam que recebem EPl's adequados e $40 \%$ recebem às vezes, não houve quem não recebe 0 material durante o plantão. Sobre a reanimação cardiopulmonar, $96,7 \%$ responderam que participam durante a conduta e 3,3\% participam às vezes, não houve quem não participasse.

Tabela 3 - Informações sobre a realização e colaboração na assistência do fisioterapeuta.

\begin{tabular}{lcccccc}
\hline \multirow{2}{*}{ Perguntas } & \multicolumn{2}{c}{ Sim } & \multicolumn{2}{c}{ Ás vezes } & \multicolumn{2}{c}{ Não } \\
\cline { 2 - 7 } & $\mathbf{N}$ & $\%$ & $\mathbf{N}$ & $\%$ & \multicolumn{1}{c}{$\mathbf{N}$} & $\%$ \\
\hline Participação durante o procedimento de IOT & 29 & 96,7 & 1 & 3,3 & - & - \\
\hline Participação durante a reanimação cardiopulmonar & 29 & 96,7 & 1 & 3,3 & - & - \\
\hline Realização de fisioterapia motora & 20 & 66,7 & 10 & 33,3 & - & - \\
\hline Realização de fisioterapia respiratória & 29 & 96,7 & 1 & 3,3 & - & - \\
\hline Recebimento de EPI's adequados & 18 & 60 & 12 & 40 & - & - \\
\hline
\end{tabular}

Legenda: * Intubação orotraqueal.

Fonte: Carvalho ES e Kundsin A, 2020.

Gráfico 1 - Assistência fisioterapêutica.

\section{Motivos para realizar a fisioterapia às vezes}

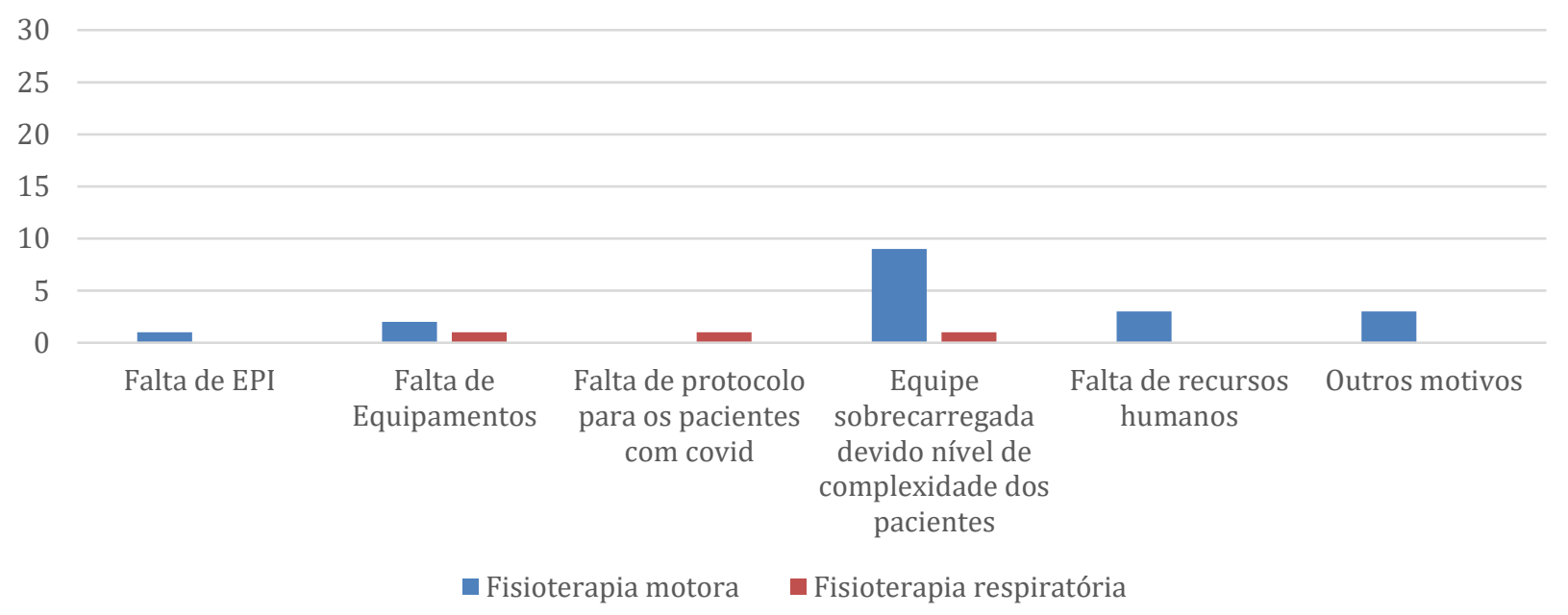

Fonte: Carvalho ES e Kundsin A, 2020. 
Durante os plantões, no quesito manejo da VMI, 3,3\% responderam que os médicos realizam este manejo, $80 \%$ responderam que são os fisioterapeutas que realizam, $6,7 \%$ responderam que ambos realizam e $10 \%$ relataram que é o médico, o fisioterapeuta e o enfermeiro. No manejo da VNI, 96,7\% responderam ser o fisioterapeuta, $3,3 \%$ relatou que é o médico que realiza e não foi obtido respostas para enfermeiro. Em relação à parada cardiorrespiratória, responderam que os ajustes da VMI são realizados $90 \%$ por fisioterapeuta, 3,3\% médico e fisioterapeuta, $6,7 \%$ responderam que é o fisioterapeuta e o enfermeiro. O manejo da oxigenoterapia $80 \%$ é por fisioterapeutas, $6,7 \%$ responderam ser o médico, fisioterapeuta e o enfermeiro, $13,3 \%$ responderam que são os fisioterapeutas e enfermeiros (Tabela 4).

Tabela 4 - Distribuição de assistências profissionais.

\begin{tabular}{lcc}
\hline Variáveis & N & $\%$ \\
\hline Realização do manejo da VMI por categoria profissional & 1 & 3,3 \\
\hline Médico & 24 & 80 \\
Fisioterapeuta & 2 & 6,7 \\
Médico e fisioterapeuta & 3 & 10 \\
Médico, fisioterapeuta e enfermeiro & 29 \\
\hline Realização do manejo da VNI ${ }^{\star}$ por categoria profissional & 96,7 \\
\hline Fisioterapeuta & 1 & 3,3 \\
Médico, fisioterapeuta e enfermeiro & 27 \\
\hline Realização de ajustes ventilatórios durante PCR ${ }^{\star *}$ por categoria profissional & 90 \\
\hline Fisioterapeuta & 1 & 3,3 \\
Médico e fisioterapeuta & 2 & 6,7 \\
Fisioterapeuta e enfermeiro & \\
\hline Realização do manejo da oxigenoterapia por categoria & 24 & 80 \\
\hline Fisioterapeuta & 2 & 6,7 \\
Médico, fisioterapeuta e enfermeiro & 4 & 13,3 \\
Fisioterapeuta e enfermeiro & \\
\hline Utilização de protocolo de mobilização precoce para pacientes com COVID-19 pelos & 21 \\
fisioterapeutas & 2 & 70 \\
\hline Sim & 7 & 23,3 \\
Não & 7 \\
Desconhece protocolos & 2020.
\end{tabular}

Legenda: *Ventilação não invasiva. ${ }^{* *}$ Parada cardiorrespiratória. Fonte: Carvalho ES e Kundsin A, 2020.

\section{DISCUSSÃO}

Nesta pesquisa, observou se que a maioria dos participantes são do gênero masculino, com predominância de fisioterapeutas jovens, com pouco tempo de formação acadêmica, porém, com experiências relatadas, tendo apenas $16,7 \%$ com formação maior que 10 anos, nesse contexto, torna-se compreensivo o grau acadêmico em maior destaque ser o de especialista.

Em partes, este resultado é contrário ao estudo de Badaró AFV e Guilhem D (2011) que investigou o perfil sociodemográfico e profissional de fisioterapeutas na cidade de Santa Maria - RS, quando comparado a predominância do gênero na área de fisioterapia, onde o dominante do estudo foi o gênero feminino. Os autores relatam que esta dominância dentro da fisioterapia, pode ser justificada devido a área exigir alta sensibilidade e atenção, geralmente, características destaque no gênero feminino. Entretanto, corrobora-se que a fisioterapia é desenhada por um perfil de profissionais jovens, que buscam conhecimentos através da especialização.

Rotta BP, et al. (2018), relatam a importância da atuação dos fisioterapeutas brasileiros dentro da UTI, apontando que a assistência fisioterapêutica ininterrupta proporciona uma assistência de maior qualidade ao paciente, otimizando a manutenção e reabilitação da função motora e respiratória, reduzindo o tempo de internação e consequentemente redução dos custos hospitalares. Furtado MVC, et al. (2020) apontam durante seu estudo, que os pacientes expostos a internações dentro do ambiente hospitalar são vulneráveis a alterações dos sistemas musculoesquelético e cardiorrespiratório, logo então, salienta que a atuação dos 
fisioterapeutas otimiza a recuperação do doente e melhora a qualidade de vida durante a internação e após a alta hospitalar.

Os achados desta pesquisa fortificam os resultados descritos por Rotta BP, et al. (2018) sobre a importância destes profissionais perante o atendimento do doente crítico. Através desta pesquisa, foi possível observar que a assistência fisioterapêutica dentro das UTl's e enfermaria do hospital é contínua, onde 76,7\% dos seus colaboradores prestam assistência por $24 \mathrm{~h} /$ dia.

Através dos resultados obtidos na distribuição de frequências das assistências fisioterapêuticas diante dos pacientes infectados por COVID-19, verifica-se que os profissionais receberam treinamentos para o manejo destes pacientes, realizando atendimento para manutenção e reabilitação do sistema motor e respiratório, conforme descrito na Tabela 2. Apesar de estarem enfrentando um momento globalmente crítico no sistema de saúde perante um vírus novo, desconhecido, com alto índice de transmissão e contaminação da população, atualmente com poucas evidências sobre o manejo dos infectados e as repercussões a curto e longo prazo, sofrem com a falta de recursos humanos, equipamento de proteção individual, recursos materiais, entre outros.

Diante de tal calamidade da saúde pública perante a situação pandêmica, a ASSOBRAFIR desde o início da pandemia do coronavírus, tem realizado publicações cientificas para nortear e capacitar os profissionais fisioterapeutas no manejo destes pacientes, tanto na assistência dos pacientes com sintomas leves, moderados e graves quanto na proteção individual dos profissionais (MATTE DL, et al., 2020).

Através desta pesquisa, é possível observar que todos os fisioterapeutas participam durante a IOT e durante a ressuscitação cardiopulmonar (RCP). Com relação ao manejo da VMI, $80 \%$ destes profissionais relataram que durante o plantão é o fisioterapeuta que realiza o manejo, porém, vale ressaltar que 3,3\% relatou ser somente o médico, $6,7 \%$ relataram ser o médico e o fisioterapeuta e $10 \%$ relataram ser o médico, fisioterapeuta e o enfermeiro. No manejo da $\mathrm{VNI}$, foi observado que $96,7 \%$ é realizado por fisioterapeutas, apenas 3,3\% relataram ser o médico e o fisioterapeuta, não houve resultados para enfermeiros neste quesito. No que se refere a administração da oxigenoterapia suplementar, $80 \%$ responderam que é o fisioterapeuta que realiza o manejo, $13,3 \%$ responderam ser o fisioterapeuta e o enfermeiro, apenas $6,7 \%$ responderam ser o médico, enfermeiro e fisioterapeuta. Com relação ao protocolo de mobilização precoce neste perfil de pacientes, $70 \%$ relataram que utilizam sendo que $23,3 \%$ relatou não conhecer nenhum protocolo.

A taxa de internações de paciente infectados por SARS-CoV-2 é crescente, tornando-se importante a atualização e capacitação dos profissionais atuantes no manejo destes indivíduos e ao cuidado individual conforme a orientação da OMS. O fisioterapeuta possui um papel importante em todas as fases de tratamento destes pacientes, gerencia a ventilação espontânea, a invasiva e a não invasiva, a oferta de oxigênio suplementar, atua no processo pré e após a IOT, na fase de extubação, a aplicação de estratégias de exercícios e mobilização precoce, na avaliação e tratamento das disfunções respiratórias, levando sempre em consideração as indicações e contraindicações de tais assistências (MUSUMECI MM, et al., 2020; SILVA VZM, et al., 2020; MARTINEZ BP e ANDRADE FMD, 2020).

A infecção da COVID-19 pode desencadear, a síndrome do desconforto respiratória agudo (SDRA) entre outros acometimentos. A SDRA é classificada em leve, moderada e grave. Um dos recursos destaque para o tratamento desta síndrome, quando se encontra no quadro moderado ou grave, é a realização da posição prona, que consiste em posicionar o paciente em decúbito ventral. Tal posicionamento tem se tornado um grande aliado no manejo dos pacientes acometidos pela covid-19, requer experiência e capacitação da equipe multidisciplinar, vale salientar que a prona deve ser associada a ventilação mecânica protetora, e possui suas indicações e contraindicações (BORGES DL, et al., 2020; JOCHMANS S, et al., 2020). Nesta pesquisa, 96,7\% dos colaboradores utilizam este posicionamento durante a assistência dos pacientes infectados, entretanto, apenas $36,7 \%$ possuíam experiências antes da pandemia.

Dos profissionais que participam deste estudo, $33,3 \%$ responderam que já testaram positivo para a covid19 sendo que $66,7 \%$ responderam que não se infectaram. No estado de Rondônia, até o dia 13 de janeiro de 2021, foram registrados 104.737 casos confirmados, sendo que $83,24 \%$ não se encontram com o vírus ativo, 
$14,88 \%$ se encontram com a infecção ativa e 1,88\% que equivale à 1.966 evoluíram para o óbito. Aguardando o resultado de exames para covid-19, possuí 1.458 indivíduos. Referente aos dados dos casos confirmados, um total de 5.861 são dos profissionais da área de saúde, destes, $87,53 \%$ não se encontram mais com o vírus ativo. $O$ estado se encontra com aumento dos casos e alta taxa de ocupação dos leitos direcionados para os pacientes com covid-19 (AGEVISA SESAU RO, 2021).

\section{CONCLUSÃO}

A COVID-19 proporcionou novos desafios e oportunidades para os fisioterapeutas, com o aumento da demanda de pacientes internados, foi necessário o aumento de profissionais. Entretanto, foi possível observar que estes profissionais sofrem com a falta de recursos humanos e EPI's, tendo risco de exposição e contaminação diariamente. Com base neste estudo, o fisioterapeuta vem atuando na condução do suporte ventilatório invasivo e não invasivo, manejo da oxigenoterapia e da posição prona, realizando exercícios com ênfase na otimização e reabilitação da função motora e respiratória, auxiliando em procedimentos corriqueiros dos cuidados intensivos como a intubação orotraqueal, extubação, reanimação cardiopulmonar. Vale ressaltar, a importância de haver o desenvolvimento de mais estudos para esta temática apresentada.

\section{REFERÊNCIAS}

1. AGEVISA SESAU RO. Sistema de comandos de incidentes - COVID-19: Sala de situações integradas; relatório de ações. Governo do Estado de Rondônia, 2021; e285.

2. BADARÓ AFV, GUILHEM D. Perfil sociodemográfico e profissional de fisioterapeutas e origem das suas concepções sobre ética. Fisioterapia em Movimento, 2011; 24 (3): 445-454.

3. BORGES DL, et al. Posição prona no tratamento da insuficiência respiratória aguda na COVID-19. ASSOBRAFIR Ciência, 2020; 11 (Supl 1): 111-120.

4. BRASIL. Ministério da Saúde. Guia prático de gestão em saúde no trabalho para covid-19. Brasília, DF, 2020.

5. BRASIL. Ministério da Saúde. Orientações para manejo de pacientes com covid-19. Brasília, DF, 2020.

6. FURTADO MVC, et al. O papel da fisioterapia no ambiente hospitalar. Pubsaúde, 2020; 4: a052.

7. GORBALENYA AE, et al. Severe acute respiratory syndrome-related coronavirus: The species and its viruses-a statement of the Coronavirus Study Group. BioRxiv, 2020.

8. GRASSELLI G, et al. Risk factors associated with mortality among patients with COVID-19 in intensive care units in Lombardy, Italy. JAMA, 2020; 180 (10): 1345-1355.

9. GUIMARÃES F. Atuação do fisioterapeuta em unidades de terapia intensiva no contexto da pandemia de COVID-19. Fisioter. Mov., 2020; 33: e0033001.

10. INSTITUTO BUTANTAN. Vacina contra a covid-19. Instituto Butantan; 2021.

11. JOCHMANS S, et al. Duration of prone position sessions: a prospective cohort study. Annals of Intensive Care, 2020; $10(66): 1-9$.

12. MARTINEZ BP, ANDRADE FMD. Estratégias de mobilização e exercícios terapêuticos precoces para pacientes em ventilação mecânica por insuficiência respiratória aguda secundária à COVID-19. ASSOBRAFIR Ciência, $2020 ; 11$ (Supl 1): 121-131.

13. MATTE DL, et al. ASSOBRAFIR reforça sua missão no enfrentamento à pandemia da COVID-19. ASSOBRAFIR Ciência, 2020; 11 (Supl 1-Série especial COVID-19): 11-13.

14. MATOS CA, et al. Existe diferença na mobilização precoce entre os pacientes clínicos e cirúrgicos ventilados mecanicamente em UTI?. Fisioter. Pesqui., 2016; 23 (2): 124-128.

15. MUSUMECI MM, et al. Recursos fisioterapêuticos utilizados em unidades de terapia intensiva para avaliação e tratamento das disfunções respiratórias de pacientes com COVID-19. ASSOBRAFIR Ciência, 2020; 11 (Supl 1): $73-$ 86.

16. NORONHA KVMS, et al. Pandemia por COVID-19 no Brasil: análise da demanda e da oferta de leitos hospitalares e equipamentos de ventilação assistida segundo diferentes cenários. Cadernos de Saúde Pública, $2020 ; 36$ (6): e00115320.

17. SANTANA G, et al. Infecção e óbitos de profissionais da saúde por COVID-19: revisão sistemática. Acta Paulista de Enfermagem, 2020; 33: eAPE20200107.

18. SILVA VZM, et al. Recomendações para a utilização de oxigênio suplementar (oxigenoterapia) em pacientes com COVID-19. ASSOBRAFIR Ciência, 2020; 11 (Supl 1): 87-91.

19. SUN P, et al. Understanding of COVID-19 based on current evidence. Journal of medical virology, $2020 ; 92$ (6): 548 551

20. WANG F. Long-term respiratory and neurological sequelae of COVID-19. Medical science monitor: international medical journal of experimental and clinical research, 2020; 26: e928996-1.

21. WIERSINGA WJ, et al. Pathophysiology, Transmission, Diagnosis, and Treatment of Coronavirus Disease 2019 (COVID-19): A Review. JAMA, 2020; 324 (8):782-793.

22. ROTTA BP, et al. Relação entre a disponibilidade de serviços de fisioterapia e custos de UTI. Jornal Brasileiro de Pneumologia, 2018; 44 (3): 184-189. 